\title{
Conductance measurement of spin-orbit coupling in the two-dimensional electron systems with in-plane magnetic field
}

\author{
K. Kolasiński, ${ }^{1,2}$ H. Sellier, ${ }^{2}$ and B. Szafran ${ }^{1}$ \\ ${ }^{1}$ AGH University of Science and Technology, Faculty of Physics and Applied Computer Science, \\ al. Mickiewicza 30, 30-059 Kraków, Poland \\ ${ }^{2}$ Institut Néel, CNRS and Université Joseph Fourier, \\ BP 166, 38042 Grenoble, France
}

\begin{abstract}
We consider determination of spin-orbit ( $\mathrm{SO}$ ) coupling constants for the two-dimensional electron gas from measurements of electric properties in rotated in-plane magnetic field. Due to the SO coupling the electron backscattering is accompanied by spin precession and spin mixing of the incident and reflected electron waves. The competition of the external and SO-related magnetic fields produces a characteristic conductance dependence on the in-plane magnetic field value and orientation which, in turn, allows for determination of the absolute value of the effective spin-orbit coupling constant as well as the ratio of the Rashba and Dresselhaus SO contributions.
\end{abstract}

Introduction. Charge carriers in semiconductor devices are subject to spin-orbit (SO) interactions [1] stemming from the anisotropy of the crystal lattice and/or the device structure. The SO interactions translate the carrier motion into an effective magnetic field leading to carrier spin relaxation and dephasing [2-4], spin Hall effects [57], formation of topological insulators [8], persistent spin helix states [9-11], Majorana fermions [12]. Moreover, the SO coupling paves the way to spin-active devices, including spin-filters based on quantum point contacts (QPCs) [13] or spin transistors [14-18], which exploit the precession of the electron spin in the effective magnetic field [19]. The most popular playground for studies of spin effects and construction of spin-active devices is the two-dimensional electron gas (2DEG) confined at an interface of an asymmetrically doped III-V heterostructure, with a strong built-in electric fields in the confinement layer giving rise to the Rashba SO coupling [20] and with the Dresselhaus coupling due to the anisotropy of the lattice which is enhanced by a strong localization of the electron gas in the growth direction [21].

The SO interaction is sample-dependent and its characterization is of a basic importance for description of spin-related phenomena and devices. The SO coupling constant are derived from the Shubnikov-de Haas [2229] oscillations, antilocalization in the magnetotransport [30], photocurrents [31], or precession of optically polarized electron spins as a function of their drift momentum [32]. Usually both the Rashba and Dresselhaus interactions contribute to the overall SO coupling. Separation of contributions of both types of SO coupling is challenging and requires procedures based on optical polarization of the electron spins [31-33]. In this Letter we investigate the possibility for extraction of the Rashba and Dresselhaus constants from a purely electric measurement of the two-terminal conductance. The proposed method does not involve application of optical excitation $[31,32]$ or a particularly complex gating [32]. The procedure given below requires rotation of the sample in an external in-plane magnetic field [34], which is straightforward as compared to application of the rotated electric field to 2DEG [32]. Also, the present approach is suitable for high mobility samples and goes without analysis of the localization effects in the magnetotransport [30].

The procedure which is proposed below bases on an idea that the effects of the SO coupling related to the wave vector component in the direction of the current flow can be excluded by a properly oriented external inplane magnetic field. The procedure exploits spin effects of backscattering - due to intentionally introduced potentials - or simply to intrinsic imperfections within the sample. In particular we show that the linear conductance of a disordered sample reveals an oscillatory behavior as a function of the magnetic field direction and amplitude. The dependence allows one to determine the strength of the SO interaction as compared to the spin Zeeman effect as well as the relative strength of both Rashba and Dresselhaus contributions.

Spin-dependent scattering model. Let us start from a simple model of electron scattering (see Fig. 1). The electron is injected to the system from e.g. a QPC and comes to the potential defect from the left. The defect is taken as an infinite potential step, so that the scattering probability is 1 . The incident and backscattered waves are denoted by $\left|k_{\sigma}^{+}\right\rangle$where $k_{\sigma}^{ \pm}$stands for the absolute value of the wave vector for the spin state $\sigma$ and the superscript sign indicates the electron incoming from the left $(+)$ or backscattered $(-)$. Only the backscattering which returns the carriers to the QPC can alter the conductance, so we consider the scattering wave function along the line between the QPC and the defect

$$
\left|\Psi_{\sigma}\right\rangle=e^{i k_{\sigma}^{+} r}\left|k_{\sigma}^{+}\right\rangle+\Sigma_{\sigma^{\prime}} a_{\sigma \sigma^{\prime}} e^{-i k_{\sigma^{\prime}}^{-} r}\left|k_{\sigma^{\prime}}^{-}\right\rangle,
$$

where $a_{\sigma \sigma^{\prime}}$ stand for the scattering amplitudes.

Scattering at other angles does not decrease the conductance and is neglected for a moment. Within the 2DEG, outside the scattering center and the QPC channel the 2D electron Hamiltonian for in-plane field $\mathbf{B}=$ 


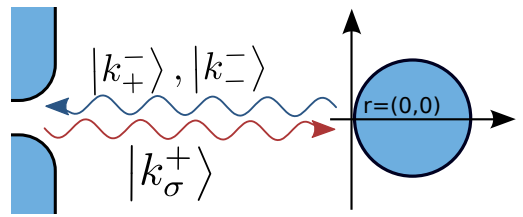

Figure 1. Sketch of considered scattering process. The electron wave is incoming from the left from a source (a QPC for instance) in spin state $\sigma$, propagates to the right and is backscattered at position $r=(0,0)$ by the potential barrier induced by the impurity.

\section{$\left(B_{\mathrm{x}}, B_{\mathrm{y}}, 0\right)$ reads}

$$
H=E_{\mathrm{kin}} \mathbf{I}+\sigma_{\mathrm{x}}\left(\alpha k_{\mathrm{y}}-\beta k_{\mathrm{x}}+b_{\mathrm{x}}\right)+\sigma_{\mathrm{y}}\left(\beta k_{\mathrm{y}}-\alpha k_{\mathrm{x}}+b_{\mathrm{y}}\right),
$$

where $\boldsymbol{E}_{\text {kin }}=\frac{\hbar^{2} \boldsymbol{k}^{2}}{2 m_{\mathrm{eff}}}, b_{\mathrm{x} / \mathrm{y}}=\frac{1}{2} g \mu_{\mathrm{B}} B_{\mathrm{x} / \mathrm{y}}, m_{\text {eff }}$ is the electron effective mass, $\alpha$ and $\beta$ are the Rashba and Dresselhaus constants. The spin Zeeman effect is introduced via Pauli matrices $\sigma_{\mathrm{x} / \mathrm{y}}$ and the Zeeman energy will be denoted below by $E_{B}=\frac{1}{2} g \mu_{\mathrm{B}}|B|=\sqrt{b_{x}^{2}+b_{y}^{2}}$.

Note, that we use the symmetric gauge $\boldsymbol{A}=$ $\left(B_{y} z,-B_{x} z, 0\right)$ then by choosing the plane of the $2 \mathrm{DEG}$ confinement to be located at $z=0$, we get $\boldsymbol{A}=\mathbf{0}$, and the magnetic field enters the Hamiltonian only via the spin Zeeman term i.e. the orbital effects do not affect the electron transport.

Let us first neglect the Dresselhaus coupling $(\beta=0)$. Plane wave solution for the eigenvalues of the Schrödinger equation gives

$$
E_{\sigma}=\frac{\hbar^{2} \boldsymbol{k}^{2}}{2 m_{\mathrm{eff}}}+\sigma|\boldsymbol{p}|,
$$

with $\sigma=\{+,-\}$ denoting projections of the spin on the direction of polarization $\boldsymbol{p}=\left(\alpha k_{\mathrm{y}}+b_{\mathrm{x}},-\alpha k_{\mathrm{x}}+b_{\mathrm{y}}\right)$, and eigenvectors

$$
\left|k_{\sigma}^{ \pm}\right\rangle=\frac{1}{\sqrt{2}}\left(\begin{array}{c}
1 \\
\sigma \frac{p_{\mathrm{x}}^{ \pm}+i p_{\mathrm{y}}^{ \pm}}{p^{ \pm}}
\end{array}\right) \equiv \frac{1}{\sqrt{2}}\left(\begin{array}{c}
1 \\
\sigma e^{i \phi\left(\boldsymbol{k}_{\sigma}^{ \pm}, \boldsymbol{B}\right)}
\end{array}\right),
$$

for the incident $(+)$ and backscattered $(-)$ directions of the electron motion with $p^{ \pm}=\left|\boldsymbol{p}^{ \pm}\right|$. Due to the assumed infinite scattering potential, the wave function in Eq. (1) has to vanish at $r=0$ (see Fig. 1), $\Psi_{\sigma}(r=0)=\left|k_{\sigma}^{+}\right\rangle+$ $\Sigma_{\sigma^{\prime}} a_{\sigma \sigma^{\prime}}\left|k_{\sigma^{\prime}}^{-}\right\rangle=0$, hence

$$
a_{\sigma \sigma^{\prime}}=-\sigma^{\prime} \frac{\sigma e^{i \phi\left(\boldsymbol{k}_{\sigma}^{+}, \boldsymbol{B}\right)}+\sigma^{\prime} e^{i \phi\left(\boldsymbol{k}_{-\sigma^{\prime}}^{-} \boldsymbol{B}\right)}}{e^{i \phi\left(\boldsymbol{k}_{+}^{-}, \boldsymbol{B}\right)}+e^{i \phi\left(\boldsymbol{k}_{-}^{-}, \boldsymbol{B}\right)}} .
$$

In the following we use $\mathrm{In}_{0.5} \mathrm{Ga}_{0.5}$ As material parameters with $m=0.0465 m_{0}$, Landé factor $g=9$, and the Fermi energy $E_{\mathrm{F}}=20 \mathrm{meV}$. For the bulk Rashba [35] constant $\alpha_{3 D}=57.2 \AA^{2}$, the $2 \mathrm{D}$ value is $\alpha=\alpha_{3 D} F_{z}$, where $F_{z}$ is the electric field in the growth-direction. The Rashba constant can be controlled by the external voltages [22] and for $\mathrm{In}_{0.5} \mathrm{Ga}_{0.5}$ As $\mathrm{SO}$ coupling constants of the order of 5 to $10 \mathrm{meV} \mathrm{nm} \mathrm{[22]} \mathrm{were} \mathrm{recorded.}$

In Fig. 2(a) we present the scattering amplitudes $a_{\sigma \sigma^{\prime}}$ obtained from Eq. (5) as a function of the direction of the magnetic field $\boldsymbol{B}=(B \cos (\theta), B \sin (\theta))$, with $B=5 \mathrm{~T}$ for scattering along the $x$ direction, $\boldsymbol{k}=\left(k_{x}, 0\right)$. Note that for the magnetic field oriented in the $y$ direction $\theta=\pi / 2$, i.e. for $B=\left(0, B_{\mathrm{y}}\right)$ the diagonal elements of the scattering amplitudes are zero. This is a special case for which the spinor in Eq. (4) can be written in form $\left|k_{\sigma}^{ \pm}\right\rangle=\left(\begin{array}{c}1 \\ i \sigma d_{ \pm}\end{array}\right)$, where $d_{ \pm}=\operatorname{sign}\left(-\alpha \mathrm{k}_{\mathrm{x}}^{ \pm}+\mathrm{b}_{\mathrm{y}}\right)$. For a weak magnetic field $\left|\alpha k_{\mathrm{x}}^{ \pm}\right|>\left|b_{\mathrm{y}}\right|$, we get $d_{ \pm}=\mp$, and the orthogonality relation $\left\langle k_{\sigma^{\prime}}^{d^{\prime}} \mid k_{\sigma}^{d}\right\rangle=\frac{1}{2}\left(1+\sigma \sigma^{\prime} d d^{\prime}\right)$, gives zero for the backscattering to states with the same spin projection on the polarization vector $(\mathbf{p}),\left\langle k_{\sigma}^{-} \mid k_{\sigma}^{+}\right\rangle=$ 0 [see Fig. 2(a)]. On the other hand for high magnetic field $\left|\alpha k_{\mathrm{x}}^{ \pm}\right|<\left|b_{\mathrm{y}}\right|$, we get $d_{ \pm}=1$, and the spin projection on the polarization vector is conserved $\left\langle k_{\sigma}^{-} \mid k_{\sigma}^{+}\right\rangle=1$.

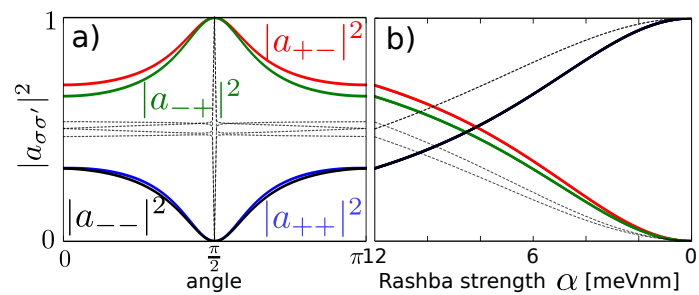

Figure 2. (a) Scattering amplitudes calculated from Eq. (5) for $\alpha=12 \mathrm{meVnm}$ and $\beta=0$ as a function of angle formed by the magnetic field vector and the $x$ axis $\theta$. (b) Same as in (a), but for a fixed angle $\theta=\pi$ as a function of Rashba constant $\alpha$. In (a) and (b) the solid lines show the result for $B=5 \mathrm{~T}$ and the black dashed lines for $B=7.2 \mathrm{~T}$.

In Fig. 2(b) we show the evolution of the scattering amplitudes for the orientation of magnetic field fixed at $\theta=\pi$, as a function of the Rashba constant $\alpha$. Then, in Eq. (2) $k_{y}=0$ and $b_{y}=0$. The scattering amplitudes cross near $\alpha \approx 8 \mathrm{meVnm}$ [Fig. 2(b)]. In this point the Zeeman energy $E_{B}$ is equal to the SO coupling energy $E_{S O}=\alpha k_{x}$. For $\alpha k_{x}=E_{B}$ the off-diagonal terms are: $-b_{x} \sigma_{x}+\left(E_{B}+b_{y}\right) \sigma_{y}$, for which the scattering amplitudes (5) for eigenvectors (4) simplify to $\left|a_{\sigma \sigma^{\prime}}\right|^{2}=\frac{1}{2}$ for any $\sigma \sigma^{\prime}$ and for any in-plane orientation of $\mathbf{B}$ vector. The $E_{B} \approx$ $E_{S O}$ case is presented in Fig. 2(a) where the black dashed lines show the scattering amplitudes for $B=7.2 \mathrm{~T}$, which shows that almost complete spin mixing $\left|a_{\sigma \sigma^{\prime}}\right|^{2} \approx \frac{1}{2}$ is present for any angle.

Let us now include the Dresselhaus SO coupling. The 2D Dresselhaus constant is given by $\beta=\beta_{3 D}\left\langle k_{z}^{2}\right\rangle=$ $\beta_{3 D} \frac{\pi^{2}}{d^{2}}$, where $\beta_{3 D}$ is the bulk constant and $d$ is the width of the 2DEG confinement in the growth direction. We consider $\beta$ values from 0 to $\simeq \alpha[9,10]$. The cubic Dresselhaus interaction is neglected as a small effect [10]. In the absence of the $B$ field, for the electron incident along the $x$ direction i.e. $k_{\mathrm{y}}=0$, the polarization di- 
rection is $\boldsymbol{p}=\left(-\beta k_{\mathrm{x}},-\alpha k_{\mathrm{x}}\right)$ with the energy eigenvalues $E_{\sigma}=\frac{\hbar^{2} k_{\mathrm{x}}^{2}}{2 m_{\mathrm{eff}}}+\sigma k_{\mathrm{x}} \sqrt{\alpha^{2}+\beta^{2}}$. As a result the Dresselhaus interaction sets the direction of the electron spin polarization to $\theta=\arctan \left(\frac{\alpha}{\beta}\right)$ and increases the effective SO coupling constant to $\gamma_{\text {eff }}=\sqrt{\alpha^{2}+\beta^{2}}$.

The above conclusions can also be reached by a direct inspection of the off-diagonal part of Hamiltonian (2) for the electron transport along the $x$ direction $\left(k_{y}=0\right.$, $\left.k_{x}=k_{F}\right)$. The effective magnetic field in Eq. (2) is $\left(-\beta k_{F}+b_{x},-\alpha k_{F}+b_{y}\right)$. Both components of the effective magnetic field vanish for

$$
\tan \theta=\frac{b_{y}}{b_{x}}=\frac{\alpha}{\beta}
$$

and

$$
E_{\mathrm{B}}=\frac{1}{2} g \mu_{\mathrm{B}} B=\sqrt{b_{x}^{2}+b_{y}^{2}}=k_{F} \gamma_{\mathrm{eff}} \equiv E_{\mathrm{SO}} .
$$

For illustration we calculated the electron density at the source position - including the incident and backscattered waves using Eqs. $(1,5)$ as $\rho=\sum_{\sigma}\left\langle\Psi_{\sigma} \mid \Psi_{\sigma}\right\rangle$. The backscattering probability is roughly proportional to the electron density at the QPC [36]. The electron density - is depicted in Fig. 3(b-d) for $\alpha=12 \mathrm{meVnm}$, $\beta=0$; and Fig. $3(\mathrm{c}-\mathrm{d}) \alpha=9 \mathrm{meVnm}, \beta=8 \mathrm{meVnm}$. These values produce the same effective coupling constant $\gamma_{\mathrm{eff}} \approx 12 \mathrm{meVnm}$.

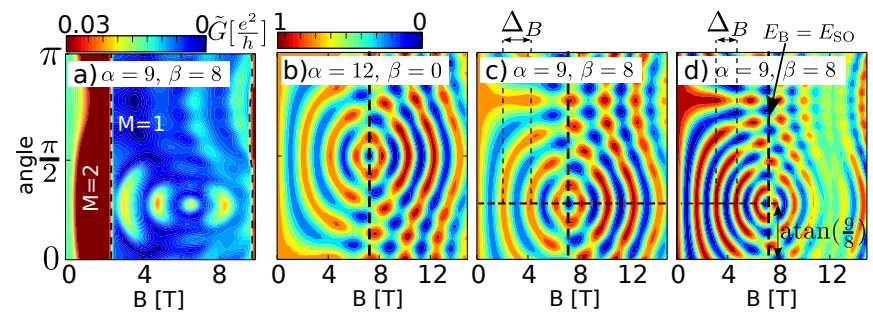

Figure 3. (a) Reduction of QPC [Fig. 1] conductance $\tilde{G}=M \frac{\mathrm{e}^{2}}{\mathrm{~h}}-G$ from its quantized value for $M$ subbands $\left[E_{F}=20 \mathrm{meV}\right]$ passing across the QPC for a potential defect at a distance of $1500 \mathrm{~nm}$ from the QPC as a function of the inplane magnetic field value and orientation. The results were calculated numerically within the Landauer approach. The QPC gate potential modeled with analytical formulas for a rectangle gate adapted from Ref. [37]. (b) Charge density at the entrance to the QPC calculated with a simple model of Eq.(5) for the QPC at $1500 \mathrm{~nm}$ from the scatterer. (c) Same as (b) but with Rashba and Dresselhaus SO interactions present. (d) Same as (c) but with the source at $2000 \mathrm{~nm}$ from the scatterer. The values of the coupling constants $\alpha$ and $\beta$ are given in $\mathrm{meV} \times \mathrm{nm}$ units. The vertical dashed line in(b-d) indicates $B=7.2 \mathrm{~T}$ for which the Zeeman energy is equal to the SO coupling energy, $E_{B}=E_{S O}$, see text. The horizontal dashed line shows the angle $\arctan \frac{\alpha}{\beta}$

The results of Fig. 3(b-d) contain a distinct circular pattern in the $\theta, B$ plane. The position of the center is given by Eqs. (6,7). The angular coordinate of the center allows one to determine the ratio of the Rashba and Dresselhaus constants and the SO coupling constant $\gamma_{\text {eff }}$ can be read-out from the position of the center of the pattern on the $B$ scale, provided that the Fermi wave vector is known. In presence of SO coupling and / or the Zeeman effect $k_{F}$ is spin dependent [21]. However, for the $E_{B}=E_{S O}$ the off-diagonal terms of the Hamiltonian (2) vanish and the Fermi wavevector is directly related to the Fermi energy $E_{F}=\frac{\hbar^{2} k_{F}^{2}}{2 m_{\text {eff }}}$, which for the adopted parameters gives $k_{F}=0.156 / \mathrm{nm}$. For $\gamma_{\text {eff }}=12 \mathrm{meV} \mathrm{nm}$ one obtains $E_{S O}=1.875 \mathrm{meV}$, which coincides with $E_{B}$ for $B=7.2 \mathrm{~T}$ (see Fig. 3(b-d)).

In Fig. 3(c) one notices a reduction of the period with respect to $3(\mathrm{~b})$ with the source-impurity distance increased to $2000 \mathrm{~nm}$ from $1500 \mathrm{~nm}$. The period of the oscillations $\Delta_{\mathrm{B}}$ is $\Delta_{\mathrm{B}}^{(c)} \approx 1.5 \mathrm{~T}$ in $(\mathrm{c})$, and $\Delta_{\mathrm{B}}^{(b)} \approx 2.0 \mathrm{~T}$ in (b). The ratio $\Delta_{\mathrm{B}}^{(c)} / \Delta_{\mathrm{B}}^{(b)} \approx 3 / 4$, is exactly an inverse of the source-impurity $d_{\mathrm{s}-\mathrm{i}}$ distance ratio.

Coherent quantum transport calculations. With the intuitions gained by the simple analytical model we can pass to the calculations of the coherent transport using a standard numerical method [38], based on the quantum transmitting boundary solution of the quantum scattering equation at the Fermi level implemented in the finite difference approach, which produces the electron transfer probability used in the Landauer formula for conductance summed over the subbands of the channels far from the scattering area. Zero temperature is assumed. For the numerical calculations we consider a channel extended along the $x$ direction, hence $k_{x}$ in Eq. (2) remains a quantum number characterizing the asymptotic states of the channel. Within the computational box the wave vector is replaced by an operator $\mathbf{k}=\left(k_{x}, k_{y}\right)=-i \nabla$.

We consider a QPC/defect system of Fig. 1. The results presented in Fig. 3(a) indicate a reduction of the conductance below the maximal value $M \frac{\mathrm{e}^{2}}{h}$ for $M$ subbands passing across the QPC. The central position of the pattern nearly coincides with the one of Fig. 3(c). The local extremum of conductance in the center of the pattern indicates the value and orientation of the external magnetic field which lifts the SO interaction effects. The angular position of Fig. 3(c) is exactly reproduced, and the amplitude of the field is $B=6.5 \mathrm{~T}$ instead of $B=7.2$ T. The deviation in the location of the central point in the $B$ axis in Fig. 3(a) results from the confinement in the QPC channel which is not included in our free particle model (see below).

The effects described so far dealt with interference of the electron waves between the source (QPC) and the defect. In fact, the role of the source can be played by any scattering center, and the extraction of the SO coupling constant requires a presence of two or more scatterers to allow for formation of standing waves described in the previous section. For the rest of the paper we 
consider a channel of homogenous width $W$, which carries $M$ transport modes at the Fermi level. In Fig. 4(d) we presented the conductance results for a clean channel of width $W=180 \mathrm{~nm}$ and the computational box of length $L=1.6 \mu \mathrm{m}$. A smooth potential barrier is introduced across the channel with height $10 \mathrm{meV}$ and width $200 \mathrm{~nm}$. Depending on the orientation of the magnetic field the number of transport modes varies between $M=17$ and $M=18$. The simulation was performed for $\alpha=9 \mathrm{meVnm}$ and $\beta=8 \mathrm{meVnm}$ as in Fig. 3(b,c). The conductance plot possesses an extremum precisely at the angle of $\theta=\arctan \frac{9}{8}$. The magnetic field of the extremum is slightly shifted to lower values than $7.2 \mathrm{~T}$ - which is a result of the reduction of $k_{x}$ within the potential barrier. The lack of conductance oscillations that were observed above in Fig. 3 results from a small barrier length $\left(d_{s-i}=200 \mathrm{~nm}\right)$.

The oscillations reappear when one replaces the barrier by a random disorder due to the random nonmagnetic and spin-diagonal potential fluctuations. The fluctuations simulate inhomogeneity of the doping of the potential barrier which provides the charge to the 2DEG. In 2DEG in III-V's due to the spatial separation of the impurities of the 2DEG, the defects do not introduce any significant contribution to the spin-orbit interaction (see Ref. [39] and the Supplement [40]). Figure 4(c) displays the conductance for the channel of the same width and length. The potential - displayed in Fig. 4(a) is locally varied within the range of $\left(-0.5 E_{\mathrm{F}}, 0.5 E_{\mathrm{F}}\right)$. The perturbation induces a multitude of scattering evens - the local density of states at the Fermi level for $B=0$ is displayed in Fig. 4(b). In spite of the complexity of the density of states the angular shift is still $\arctan (9 / 8) \approx \pi / 4$. The shift of the $G$ extremum along the $B$ scale with respect to $7.2 \mathrm{~T}$ is detectable - but small and of an opposite sign than in Fig. 4(d). This shift is related to the fact that for a finite width channel $k_{y}$ is an operator that mixes the subbands. The wave vector $k_{y}$ is a well-defined quantum number for electrons moving in an unconfined space. The small - but detectable - effects of a finite $W$ disappear completely for a wider channel - which is illustrated in Fig. 4(e) for $W=0.8 \mu \mathrm{m}$. Here, the number of conducting bands varies between 80 and 81 . The local extremum of conductance appears exactly at the positions indicated in the previous section. Note, that although the number of subbands change by 1 in Fig. 4(c,e), the variation of conductance is as large as $\sim 3 e^{2} / h$ in Fig. 4(c) and $\sim 6 e^{2} / h$ in Fig. 4(e). The conductance variation in Fig. 3(a) was very small - since the defect was far away from the QPC, for the disordered channel it is no longer the case. For completeness in Fig. 4(f) we presented calculations for a twice smaller SO coupling constants $\alpha=4.5$ $\mathrm{meVnm}, \beta=4 \mathrm{meVnm}$, and $\gamma_{\mathrm{eff}}=6 \mathrm{meVnm}$. The position of the maximal $G$ along the $B$ scale is consistently reduced from $7.2 \mathrm{~T}$ to $3.6 \mathrm{~T}$, and the orientation of the magnetic field vector corresponding to the extremum is unchanged.

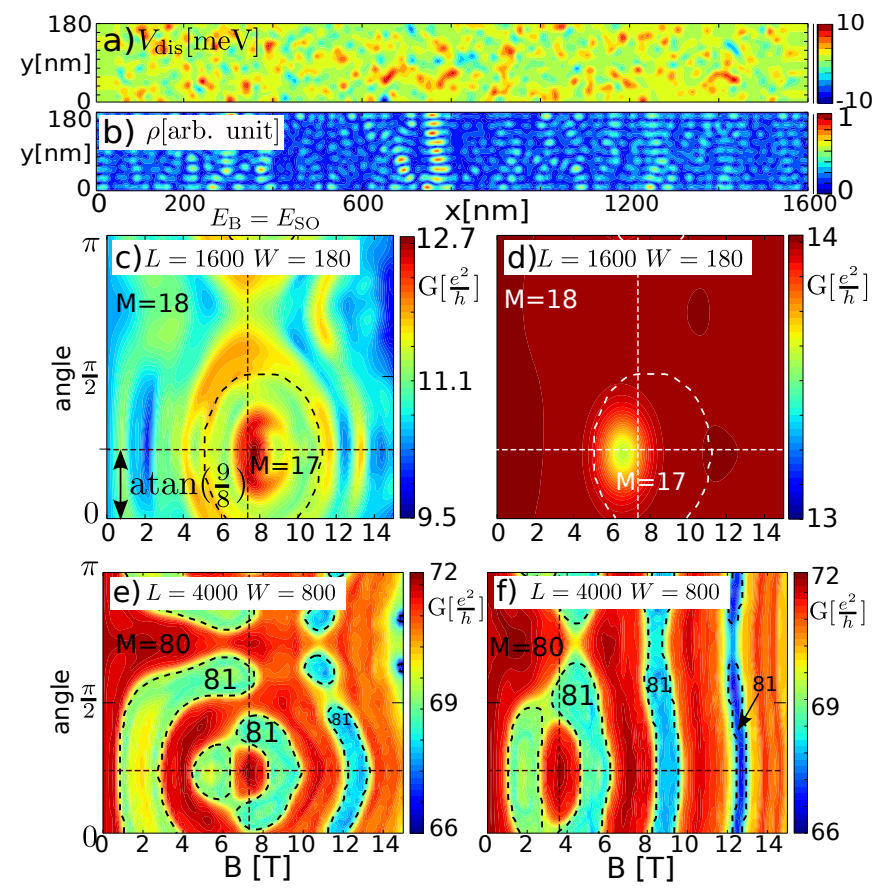

Figure 4. a) Potential disorder in simulated quantum wire. b) Local density of states obtained for channel from (a) at $\mathrm{B}=0$. c) Conductance through the wire in (a) as a function of magnetic field amplitude $B$ and direction angle. d) Same as c) but with one potential barrier in the middle of channel instead of random disorder. e) Same as (c) but for the wire with length $L=4000 \mathrm{~nm}$ and $W=800 \mathrm{~nm}$. f) Same as (e) but for SO couplings twice smaller $\gamma_{\text {eff }}=6 \mathrm{meVnm}$. For comparison of $(\mathrm{c}, \mathrm{e})$ see Fig. 3(b). The values $M$ show the number of non-degenerated modes in the channel.

Summary. We have shown that the in-plane magnetic field can lift the off-diagonal terms of the transport Hamiltonian for the two-dimensional electron gas that result from the Zeeman effect and the SO interaction. The effect appears only for a value and orientation of the external magnetic field which excludes the spin mixing effects that accompany the backscattering in presence of the SO coupling. In consequence the conductance maps for a system containing two or more scatterers - intentionally introduced - or inherently present in a disordered sample exhibit a pronounced extremum as a function of the magnetic field modulus $B$ and orientation $\theta$. An experimental value of $B$ - for which the Zeeman energy is equal to the SO coupling energy - should allow one to extract the effective SO coupling constant including both the Rashba and Dresselhaus terms, and the orientation field indicates the relative contributions of both. The results indicate the ratio of the Dresselhaus and Rashba constants is exactly resolved by the procedure, and the amplitude of the magnetic field - hence the effective SO constant varies only within a $10 \%$ from the exact value depending on the channel width and disorder profile. 
Acknowledgments This work was supported by National Science Centre (NCN) grant DEC2015/17/N/ST3/02266, and by PL-Grid Infrastructure. The first author is supported by the Smoluchowski scholarship from the KNOW funding and by the NCN Etiuda stipend DEC-2015/16/T/ST3/00310.

[1] A. Manchon, H. C. Koo, J. Nitta, S. M. Frolov, and D. R. A., Nat. Mater. 14, 871 (2015)

[2] Y. Ohno, R. Terauchi, T. Adachi, F. Matsukura, and H. Ohno, Phys. Rev. Lett. 83, 4196 (1999)

[3] M. I. D'yakonov and V. I. Perel, JETP Lett. 13, 467 (1971)

[4] J. Kainz, U. Rössler, and R. Winkler, Phys. Rev. B 70, 195322 (2004)

[5] J. E. Hirsch, Phys. Rev. Lett. 83, 1834 (1999)

[6] J. Sinova, D. Culcer, Q. Niu, N. A. Sinitsyn, T. Jungwirth, and A. H. MacDonald, Phys. Rev. Lett. 92, 126603 (2004)

[7] Y. K. Kato, R. C. Myers, A. C. Gossard, and D. Awschalom, Science 1910, 306 (2004)

[8] M. Koenig, S. Wiedmann, C. Bruene, A. Roth, H. Buhmann, L. W. Molenkamp, X.-L. Qi, and Z. S.-C., Science 318, 766 (2007)

[9] B. A. Bernevig, J. Orenstein, and S.-C. Zhang, Phys. Rev. Lett. 97, 236601 (2006)

[10] J. D. Koralek, C. P. Weber, J. Orenstein, B. A. Bernevig, S.-C. Zhang, S. Mack, and D. D. Awschalom, Nature 458, $610(2009)$

[11] M. P. Walser, C. Reichl, W. Wegscheider, and G. Salis, Nature Phys. 8, 757 (2012)

[12] V. Mourik, K. Zuo, S. M. Frolov, S. R. Plissard, E. P. A. M. Bakkers, and L. P. Kouwenhoven, Science 336, 1003 (2012)

[13] P. Debray, S. M. S. Rahman, J. Wan, R. S. Newrock, M. Cahay, A. T. Ngo, S. E. Ulloa, S. T. Herbert, M. Muhammad, and J. M., Nature Nanotech. 4, 759 (2009)

[14] S. Datta and B. Das, Appl. Phys. Lett. 56, 665 (1990)

[15] J. Schliemann, J. C. Egues, and D. Loss, Phys. Rev. Lett. 90, $146801(2003)$

[16] I. Žutić, J. Fabian, and S. Das Sarma, Rev. Mod. Phys. 76, 323 (2004)

[17] P. Chuang, S.-C. Ho, L. W. Smith, F. Sfigakis, M. Pepper, C.-H. Chen, J.-C. Fan, J. P. Griffiths, I. Farrer, H. E. Beere, G. A. C. Jones, D. A. Ritchie, and T.-M. Chen, Nat Nano 10, 35 (2015)

[18] S. Bednarek and B. Szafran, Phys. Rev. Lett. 101, 216805 (2008)

[19] L. Meier, G. Salis, I. Shorubalko, E. Gini, S. Schoen, and K. Ensslin, Nature Phys. 3 (2007)
[20] Y. Bychkov and E. Rashba, J. Phys. C 17, 6039 (1984)

[21] R. Winkler, Spin-orbit Coupling Effects in TwoDimensional Electron and Hole Systems (Springer-Verlag Berlin Heidelberg, 2003)

[22] J. Nitta, T. Akazaki, H. Takayanagi, and T. Enoki, Phys. Rev. Lett. 78, 1335 (1997)

[23] G. Engels, J. Lange, T. Schäpers, and H. Lüth, Phys. Rev. B 55, R1958 (1997)

[24] I. Lo, J. K. Tsai, W. J. Yao, P. C. Ho, L. W. Tu, T. C. Chang, S. Elhamri, W. C. Mitchel, K. Y. Hsieh, J. H. Huang, H. L. Huang, , and W.-C. Tsai, Phys. Rev. B 65 , R161306 (2002)

[25] J. H. Kwon, H. C. Koo, J. Chang, S.-H. Han, and J. Eom, Appl. Phys. Lett. 90, 112505 (2007)

[26] D. Grundler, Phys. Rev. Lett. 84, 6074 (2000)

[27] K.-H. Kim, H.-j. Kim, H. C. Koo, J. Chang, and S.-H. Han, Appl. Phys. Lett. 97, 012504 (2010)

[28] B. Das, D. C. Miller, S. Datta, R. Reifenberger, W. P. Hong, P. K. Bhattacharya, J. Singh, and M. Jaffe, Phys. Rev. B 39, 1411 (1989)

[29] Y. Ho Park, H.-j. Kim, J. Chang, S. Hee Han, J. Eom, H.-J. Choi, and H. Cheol Koo, Appl. Phys. Lett. 103, 252407 (2013)

[30] T. Koga, J. Nitta, T. Akazaki, and H. Takayanagi, Phys. Rev. Lett. 89, 046801 (2002)

[31] S. D. Ganichev, V. V. Bel'kov, L. E. Golub, E. L. Ivchenko, P. Schneider, S. Giglberger, J. Eroms, J. De Boeck, G. Borghs, W. Wegscheider, D. Weiss, and W. Prettl, Phys. Rev. Lett. 92, 256601 (2004)

[32] L. Meier, G. Salis, I. Shorubalko, E. Gini, S. Schon, and K. Ensslin, Nature Phys. 3, 650 (2007)

[33] G. Wang, B. Liu, A. Balocchi, P. Renucci, C. Zhu, T. Amand, C. Fontaine, and X. Marie, Nat. Comm. 4, $2372(2013)$

[34] S. Meckler, M. Gyamfi, O. Pietzsch, and R. Wiesendanger, Rev. Sci. Instrum. 80, 023708 (2009)

[35] E. A. de Andrada e Silva, G. La Rocca, and F. Bassani, Phys. Rev. B 55, 16293 (1997)

[36] K. Kolasiński, B. Szafran, and M. P. Nowak, Phys. Rev. B 90, 165303 (2014)

[37] J. H. Davies, I. A. Larkin, and E. V. Sukhorukov, J. Appl. Phys 77, 4504 (1995), we apply the formula for the finite rectangle gates, given by equation $V_{\mathrm{QPC}} / V_{\mathrm{g}}=g(x-L, y-$ $B)+g(x-L, T-y)+g(R-x, y-B)+g(R-x, T-y)$, where $g(u, v)=\frac{1}{2 \pi} \arctan \frac{u v}{d R} ; R=\sqrt{v^{2}+u^{2}+d^{2}}$, with $V_{g}=62 \mathrm{meV}, L=-200 \mathrm{~nm}, R=200 \mathrm{~nm}, B=-\infty$ (and $T=-62.5 \mathrm{~nm}$ for bottom gate) and $T=\infty$ (and $B=+62.5 \mathrm{~nm}$ for top gate) and $d=50 \mathrm{~nm}$. Width of the opening of the QPC is $125 \mathrm{~nm}$.

[38] K. Kolasiński, A. Mreńca-Kolasińska, and B. Szafran, Phys. Rev. B 93, 035304 (2016)

[39] P. Liu, F. Martins, B. Hackens, L. Desplanque, W. X, M. G. Pala, S. Huant, V. Bayot, and H. Sellier, Phys. Rev. B 91, 075313 (2015)

[40] K. Kolasiński, H. Sellier, and B. Szafran, Supplemental material 


\title{
Supplemental Material for Conductance measurement of spin-orbit coupling in the two-dimensional electron systems with in-plane magnetic field
}

\author{
K. Kolasiński, ${ }^{1,2}$ H. Sellier, ${ }^{2}$ and B. Szafran ${ }^{1}$ \\ ${ }^{1}$ AGH University of Science and Technology, Faculty of Physics and Applied Computer Science, \\ al. Mickiewicza 30, 30-059 Kraków, Poland \\ ${ }^{2}$ Institut Néel, CNRS and Université Joseph Fourier, \\ BP 166, 38042 Grenoble, France
}

\section{STABILITY OF THE CONDUCTANCE PATTERN}

The Letter indicated a well resolved extremum of conductance for the conditions given by Eq. (6) and (7), for which the external magnetic field cancels the SO effective magnetic field that depends on the electron wave vector. The presented analysis is based on the electron transport along the $x$ direction. The conductance extremum was found for a quantum point contact with a single defect [Fig. 3(a)] and for a potential barrier [Fig. 3(d)]. These two systems corresponded to a nearly $1 \mathrm{D}$ transport, since the potential barrier was introduced in a separable manner, and in the quantum point contact an appearance of the non-zero $k_{y}$ implies removal of the electron from the beam of backscattered electrons. However, for the perturbed sample [Fig. 4(c,e,f)] the electrons undergo multiple scattering and acquire non-zero $k_{y}$ values between the scattering events. Results of Fig. 4(c,e,f) obtained for a disorder covering basically the entire width of the sample indicate that the effects of temporarily non-zero $k_{y}$ for the spin precession averages out to zero and the conductance extremum stays in the position defined by Eqs. $(6,7)$. In order to support the discussion further we present in this document the case with a smaller number of defects, where the averaging may not be complete. We take a channel of width $W=400 \mathrm{~nm}$ and length $L=1200 \mathrm{~nm}$. The simulation was performed for $\alpha=9 \mathrm{meVnm}$ and $\beta=$ 8 meVnm as in Fig. 3(b,c). In the first column of Fig. S1 we show the obtained conductance images for different configurations of disorder potential $V_{\text {dis }}$ in the channel (second column). The third column present an image of LDOS computed for $B=0$.

One notices the angle shift $\theta=\arctan \left(\frac{\alpha}{\beta}\right)$, equal to the ratio between Rashba and Dresselhaus strengths, remains constant for different densities and configurations of disorder. Hence still for a limited number of defects the measurement of $\theta$ should provide good information about the $\frac{\alpha}{\beta}$. However, the position of the vertical line for which $E_{B}=E_{S O}$ can be estimated roughly from the obtained images to be between 7 and $8 \mathrm{~T}$ which agrees with the assumed value of the SO interaction in simulated device. The exact position of the center on the $B$ scale for the adopted parameters is $7.2 \mathrm{~T}$.

To conclude, in general we have found that detecting
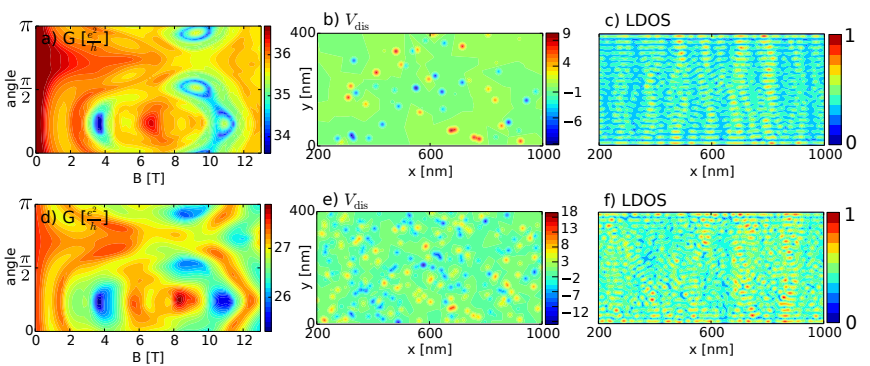

Figure S1. First column - conductance as a function of magnetic field amplitude and angle. Second column - the random disorder potential energy in the channel in units of meV. Third column - resulting LDOS for $B=0 \mathrm{~T}$ in arbitrary units.

the angle shift $\theta$ is more robust against the sample variations than finding the magnetic field strength symmetry point.

\section{THE EFFECT OF THE IMPURITIES ON SO COUPLING CONSTANT}

The disorder considered in the Letter is a potential fluctuation, which results from e.g. non-ideally homogenous doping of the barrier layer under which the twodimensional electron gas is confined. In the 2DEG heterostructures the electron gas is separated from the impurities by an undoped spacer of a few to about $20 \mathrm{~nm}$. The potential fluctuation affects the electric field in the growth direction, so an estimation of the resulting local change of the Rashba SO coupling constant is needed.

The Rashba SO interaction strength $\alpha=\alpha_{3 D} F_{z}^{\prime}$ results from the structural inversion asymmetry in the 2DEG confinement plane and is proportional to the electric field in the growth direction $F_{z}^{\prime}$, where for the bulk $\mathrm{In}_{0.5} \mathrm{Ga}_{0.5}$ As alloy Rashba [1] constant is $\alpha_{3 D}=57.2 \AA^{2}$. The strength of the electric gradient $F_{z}^{\prime}$ can be tuned by external voltages [2] and may be varied from 50 to 100 $\mathrm{kV} / \mathrm{cm}$ in $\mathrm{In}_{0.5} \mathrm{Ga}_{0.5} \mathrm{As}$ as reported in Ref. [2]. The value of the electric field can be also changed by random impurities in the structure or result from the inhomogeneity of the distribution of the impurities, which may locally change the value of $F_{z}^{\prime} \rightarrow F_{z}^{\prime}+\Delta F_{z}$. The vertical position of the 2DEG sheet is set at $z=0$. In order to estimate the strength of the electric gradient induced by 
single impurity we computed the electric field

$$
\Delta F_{\mathrm{z}}(x, y)=-\left.\frac{\partial}{\partial z}\left(\frac{e}{4 \pi \epsilon \epsilon^{*}\left|\boldsymbol{r}_{\mathrm{imp}}-\boldsymbol{r}^{\prime}\right|}\right)\right|_{\boldsymbol{r}^{\prime}=(x, y, 0)}
$$

induced by negatively charged impurity located $10 \mathrm{~nm}$ from the 2DEG surface (see Fig. S2(c)). The correction to the electric field due to a single impurity located is negligibly weak as compared to the nominal value of $F_{\mathrm{z}}^{\prime}$, hence it can be neglected in our simulations.

\section{FRIEDEL OSCILLATIONS AND THE EFFECTIVE POTENTIAL}

In strongly perturbed systems the charge density forms ripples with the periodicity of half of the Fermi wavelength. We considered the effect for the present perturbation - an impurity at a distance from the 2DEG. We simulated the effect of the single negatively charged impurity on the electron density using the DFT method described in Ref. [3]. In simulations we put $\alpha=10 \mathrm{meVnm}$ and $\beta=0$ i.e. only the Rashba term is active. The impurity is introduced $10 \mathrm{~nm}$ from the the $2 \mathrm{DEG}$ computational sheet in the middle of the computational box. The average electron density in the simulation is $1 \times 10^{12}\left[\frac{1}{\mathrm{~cm}^{2}}\right]$. The self-consistent DFT solution is reached for temperature $T=1 \mathrm{~K}$. The rest of the parameters is the same as in the transport simulations.

In Fig. S2(a) we show the self consistent electron density obtained from our DFT solver. One may see that the effect of introduced impurity is relatively weak for a given configuration. The electron density for $E_{F}=20 \mathrm{meV}$ is large, hence the screening is quite efficient, resulting in small depletion in the electron density. The change of the effective potential energy due to the presence of the defect is well visible (see Fig. S2(b)) and vary from about $42 \mathrm{meV}$ to $46 \mathrm{meV}$.

In Fig. S3(a) we plot the cross section of the electron density and effective potential from Fig. S2(a-b) with peak in the $V_{\text {eff }}$ and deep in electron density $n$ at $x=200$ nm. Due to the relatively weak perturbation of the impurity ( $\sim 25 \%$ of depletion) the amplitude of the Friedel oscillations are very small but still visible in the electron density plot. However, due to the smearing property of the Coulomb kernel $V(\boldsymbol{r})=\int \boldsymbol{d} \boldsymbol{r}^{\prime} n\left(\boldsymbol{r}^{\prime}\right) /\left|\boldsymbol{r}-\boldsymbol{r}^{\prime}\right|$ the weak Friedel oscillations in $n$ become even weaker in the resulting potential energy, hence the lack of the Friedel oscillation in the obtained $V_{\text {eff }}$ plot. In order to enhance the oscillation of the charge density in Fig. S3(b) we put the impurity at $2.5 \mathrm{~nm}$ only from the electron gas. Now, the ripples on the density are more pronounced, but their are not resolved in the self-consistent potential anyway. To conclude, the effect of Friedel oscillation on the DFT potential for the considered perturbation is weak, and the modification of the charge density by the rota-

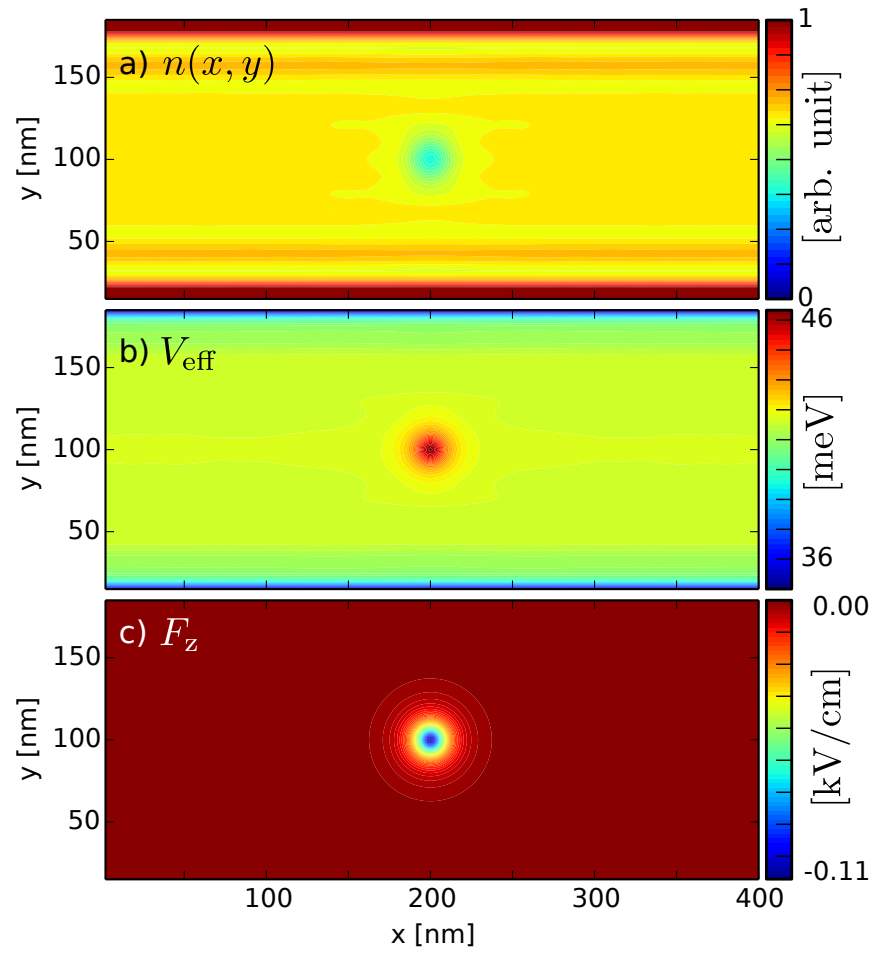

Figure S2. (a) The self consistent electron density in the channel of width $200 \mathrm{~nm}$ and length $400 \mathrm{~nm}$. (b) DFT self-consistent potential energy. (c) The bare electric field $\Delta F_{\mathrm{z}}(x, y)$ at $z=0$ induced by the impurity (see Eq. (1)).

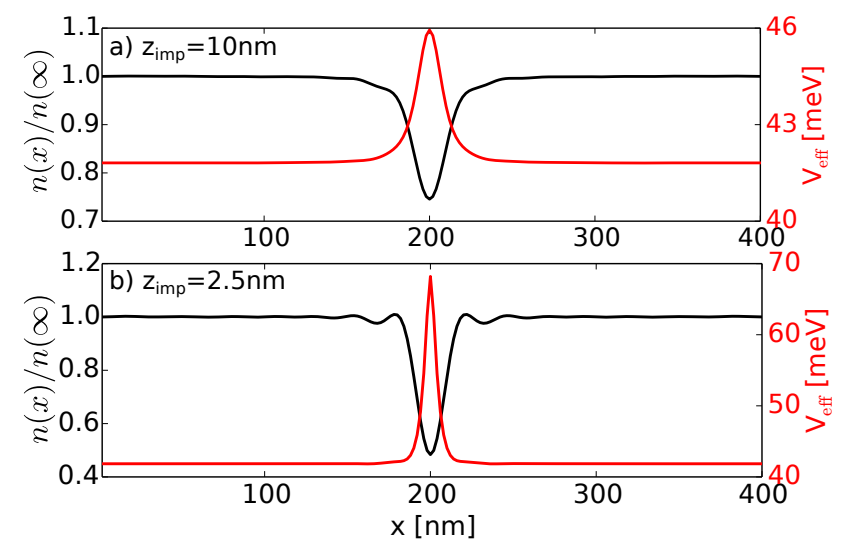

Figure S3. (a) The cross section of normalized electron density for $y=100 \mathrm{~nm}$ from Fig. S2(a) (black line) and the effective potential - potential of the impurity screened by the deformation of the electron gas (red line). (b) Same as (a) only for the impurity at $2.5 \mathrm{~nm}$ from the electron gas.

tion of the magnetic field in presence of the SO coupling should not alter the results for the electron transport.

[1] E. A. de Andrada e Silva, G. La Rocca, and F. Bassani, Phys. Rev. B 55, 16293 (1997) 
[2] J. Nitta, T. Akazaki, H. Takayanagi, and T. Enoki, Phys. Rev. Lett. 78, 1335 (1997)
[3] K. Kolasiński, A. Mreńca-Kolasińska, and B. Szafran, Phys. Rev. B 93, 035304 (2016) 